\title{
Electron localization in metal-decorated graphene
}

\author{
Wei Li, ${ }^{1}$ Yuheng He, ${ }^{2}$ Lin Wang, ${ }^{2}$ Guohui Ding, ${ }^{3}$ Zhao-Qing Zhang, ${ }^{2}$ Rolf W. Lortz, ${ }^{1,2}$ Ping Sheng, ${ }^{1,2}$ and Ning Wang ${ }^{1,2, *}$ \\ ${ }^{1}$ William Mong Institute of Nano Science and Technology, Hong Kong University of Science and Technology, Hong Kong, China \\ ${ }^{2}$ Department of Physics, Hong Kong University of Science and Technology, Hong Kong, China, \\ ${ }^{3}$ Department of Physics, Shanghai Jiaotong University, Shanghai, China
}

(Received 26 February 2011; revised manuscript received 14 May 2011; published 15 July 2011)

\begin{abstract}
By decorating single-layer graphene with disordered noble metal ( $\mathrm{Ag}, \mathrm{Au}$, and $\mathrm{Pt}$ ) clusters, we investigated experimentally the influence of strong random scatterings on graphene transport and electron-localization phenomena. As evidenced by micro-Raman scattering, there is a strong interction between the metal clusters and graphene. We found that such a strong interaction was the consequence of plasma-assisted decoration of the graphene by the metal clusters. A large negative magnetoresistance (MR) effect (up to $80 \%$ at $12 \mathrm{~T}$ ) was observed and fitted using different models. The structure, size, and area density of metal clusters were characterized by scanning tunneling microscopy and transmission electron microscopy. The samples with a high concentration of scattering centers behaved as insulators at low temperatures and showed strong localization (SL) effects. Their temperature-dependent conductance was in accordance with the two-dimensional variable-range hopping (VRH) mechanism. The localization lengths and density of states were estimated and discussed.
\end{abstract}

DOI: 10.1103/PhysRevB.84.045431

PACS number(s): 72.80.Vp, 73.20.Fz

\section{INTRODUCTION}

Owing to the hexagonal lattice structure and the attendant pseudospin quantum number, single-layer graphenes ${ }^{1}$ show quantum interference effects distinct from the conventional two-dimensional (2D) systems. In ordinary 2D metals the localization properties [either weak localization (WL) or weak antilocalization (AL)] are controlled by impurity scattering with spin-orbit interaction. ${ }^{2}$ As a result of graphene's honeycomb crystal structure, charge carriers in single-layer graphene travelling around a loop can have a Berry's phase of $\pi,^{3,4}$ and hence the coherent backscattering effect is suppressed. Electrons in graphene therefore have a strong tendency not to localize. ${ }^{5,6}$

Charge carriers in graphene reside in two nonequivalent valleys at the $\mathrm{K}$ and $\mathrm{K}^{\prime}$ points of the first Brillouin zone. In the absence of intervalley scattering, single-layer graphene should not display any WL. However, it has been reported theoretically and experimentally that WL and magnetoresistance (MR) effect in graphene can be achieved in samples with sufficiently strong intervalley scatterings. ${ }^{7-14}$ Therefore, by introducing point defects or lattice disorders, the intervalley scattering can be enhanced and constructive interference of back scattering, which favors WL, can be restored. So far the scattering mechanism, which determines the transport properties in graphene samples containing disorders, has not been unambiguously identified.

Recently some effective treatments for increasing the scattering centers in single-layer graphene have been reported, for example, by metal-element doping, ${ }^{15}$ ion bombardment, ${ }^{16}$ and chemical reaction. ${ }^{17,18}$ Hybridization of graphene by transforming partially $s p^{2}$ bonds to $s p^{3}$ bonds through hydrogenation of graphene ${ }^{19}$ can effectively cause the resistivity to diverge at low temperatures. By introducing a mild disorder into graphene for strong intervalley scattering, transition from WL to strong localization (SL) has been reported. ${ }^{20}$ Because single-layer graphene is one atom thick, chemical reaction or modification, for example, by hydrogen absorption, ${ }^{21}$ can lead to gap opening such that graphene can undergo a transition from metallic to insulating behavior. For a mild metal-doping treatment, the transport properties of graphene can be altered significantly including the decrease of mobility, shifts in the gate voltage of minimum conductivity, slight decrease of the minimum conductivity $\sigma_{\min }$ (but still remained above $e^{2} / h$ at low temperature), and plateau broadening around $\sigma_{\min }$. All these features have been predicted ${ }^{22-25}$ by the model of charged impurity or Coulomb scattering in graphene in which no backscattering interference is involved. By introducing latticedefect scattering, however, both intravalley and intervalley scatterings are expected. Backscattering interference and SL effect can result in the transition from metallic to insulating states.

In this paper we report on the study of electron-localization phenomena in metal-decorated graphene. We demonstrate that decoration by $\mathrm{Ag}$ clusters ( $\mathrm{Au}$ and $\mathrm{Pt}$ also showed similar behavior), using DC plasma deposition, can introduce random scattering centers in graphene. The Ag-cluster structures and their area density were characterized through electron microscopy observation. Strong interaction between the clusters and graphene was evidenced by Raman measurements. Upon increasing the area density of the metal clusters, the graphene devices were observed to transform from the WL to the SL regime. A large negative MR effect (up to $80 \%$ at $12 \mathrm{~T}$ ) was observed. We show that the localized states in the energy spectrum of carriers in graphene are tunable by changing the density of Ag clusters. In addition the samples containing strong scattering centers behave as insulators at low temperatures, and their transport can be described by hopping conductance in accordance with the $2 \mathrm{D}$ variable-range hopping (VRH) mechanism. The electron-localization lengths were estimated and discussed for three samples with varying area density of Ag clusters.

\section{EXPERIMENT}

High-quality graphene devices were fabricated by conventional electron-beam lithography (Raith e_LiNE). Single-layer graphene samples were prepared by mechanical exfoliation of 

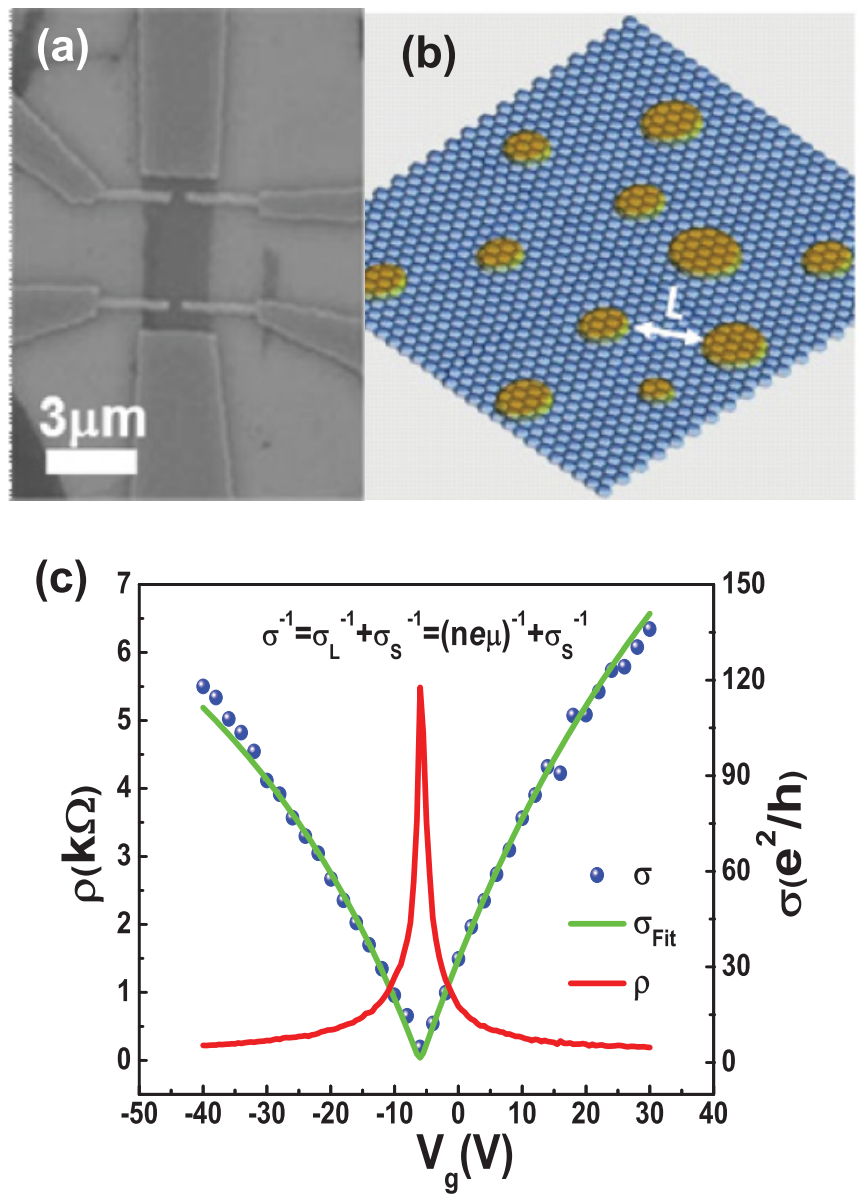

FIG. 1. (Color online) (a) A single-layer graphene device prepared by e-beam lithography. (b) Schematic drawing of the metal clusters deposited on the graphene device surface. The separation between the clusters is labeled by " $L$ " (the edge-to-edge distance) which reflects the perfect graphene area unaffected by the clusters. (c) The conductivity versus gate voltage for the pristine sample before Ag decoration.

graphite on a heavily doped $\mathrm{Si}$ substrate coated with $\mathrm{SiO}_{2}$ (300-nm thick). The electrodes were $\mathrm{Cr}(5 \mathrm{~nm}) / \mathrm{Au}(40 \mathrm{~nm})$ prepared by electron-beam thermal evaporation. The quality of the single-layer graphene samples were first verified by micro-Raman spectroscopy. The graphene devices were then characterized using four-probe configuration [Fig. 1(a)] at cryogenic temperatures (from $1.8 \mathrm{~K}$ to room temperature) before metal decoration. All transport measurements were carried out using lock-in technique (Stanford Research System SR830) with an AC current source (Keithley model 6221). The frequency of the AC-bias current $(<10 \mathrm{nA})$ was $4.7 \mathrm{~Hz}$. All graphene devices used in this study showed good homogeneity with the Dirac point close to zero back gate voltage [see Fig. 1(c)]. The conductivity at high gate regions showed nonlinearity. By considering short-range scattering, the electron mobility of the pristine graphene devices was estimated to be about $19500 \mathrm{~cm}^{2} \mathrm{~V}^{-1} \mathrm{~s}^{-1}$ and its electrical conductance at the Dirac point was about $1.18\left(4 e^{2} / h\right)$, which is close to the minimum conductance of monolayer graphene. At $2 \mathrm{~K}$ the pristine graphene device showed clear chiral quantum-Hall effect and electron mobility obtained from the
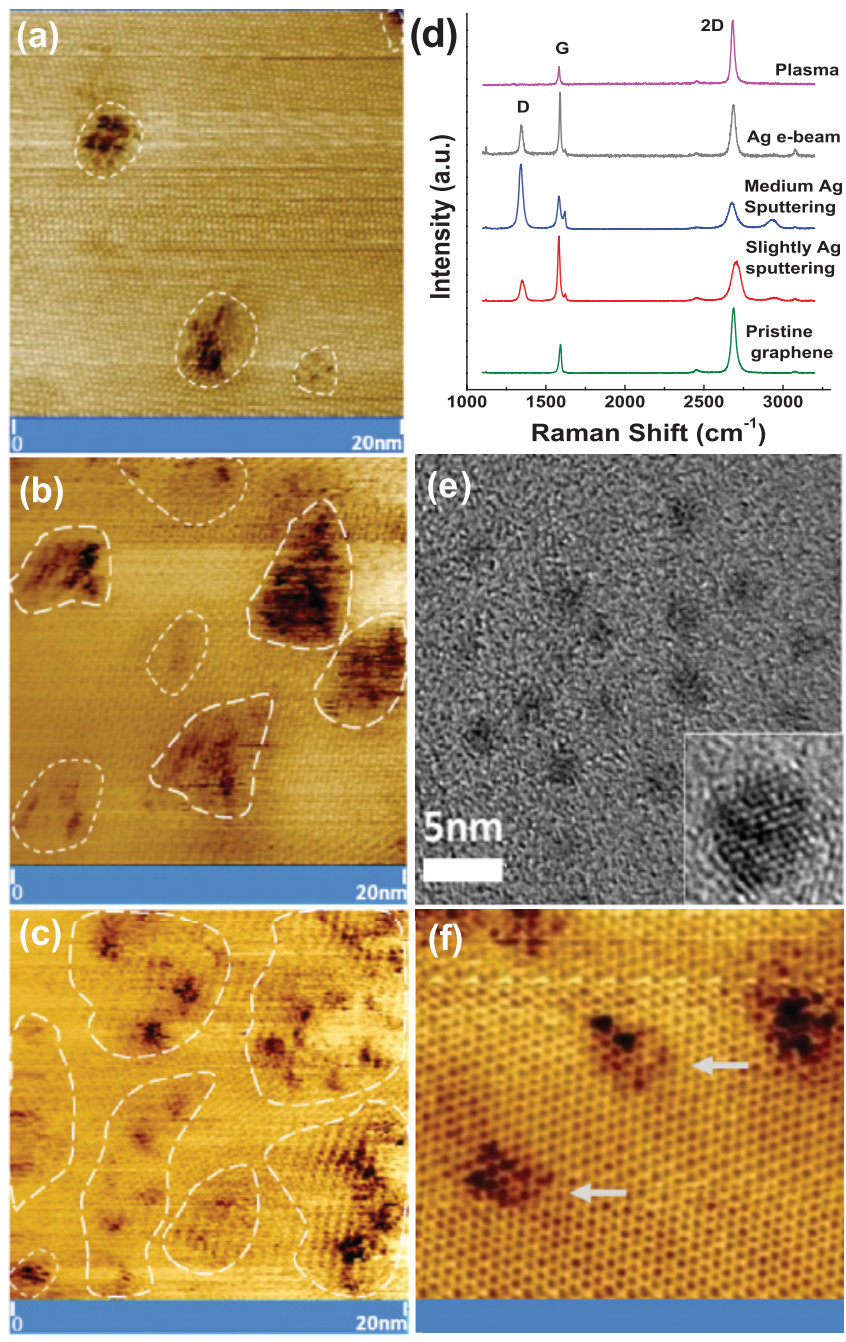

FIG. 2. (Color online) (a)-(c) STM current images (bias = $0.1 \mathrm{~V}$ ) for Ag clusters deposited on graphite surfaces for $0.5 \mathrm{~s}, 2 \mathrm{~s}$, and $10 \mathrm{~s}$, respectively. (d) Raman spectra for the pristine and Ag-decorated devices. (e) TEM images showing Ag clusters deposited on the graphene sample (2 s). (f) The high-resolution STM image of the Ag clusters distributed on the samples deposited for $5 \mathrm{~s}$.

Hall-effect measurement is close to the value mentioned above. Metal decoration of graphene devices [schematically illustrated in Fig. 1(b)] were performed in a homemade DC plasma-sputtering system with ultra-high vacuum sputtering source (model A320-UA, AJA International, USA) and DC power supply (model MDX-500, Advanced Energy, USA). A cap with a small hole was used to allow the metal-cluster flux to pass and deposit only on the graphene device. The optimum power of the DC plasma is less than $5 \mathrm{~W}$. The structure of the metal clusters was characterized by a scanning tunneling microscope (STM, Veeco Innova) and a high-resolution transmission electron microscope (HRTEM, JEOL 2010F) operated at $200 \mathrm{kV}$. For comparison the DC-plasma treatment of pristine graphene samples without metal deposition was also performed using a plasma cleaner (model PDC-002, Harrick Plasma) at a power of $7 \mathrm{~W}$. Without using plasma, metal clusters were deposited by an electron-beam evaporation system (model Peva-450E, Advanced System Technology). 


\section{RESULTS AND DISCUSSION}

Figures 2(a)-2(c) are STM images showing the morphology of $\mathrm{Ag}$ clusters deposited on different sample surfaces. The cluster density was controlled by the deposition duration (from 0.5 to 10 seconds). For lightly coated samples [Fig. 2(a), 0.5 seconds] the average separation between Ag clusters was approximately $20 \mathrm{~nm}$. The average cluster diameter is approximately $4 \mathrm{~nm}$. By increasing the deposition duration to 2 seconds (labeled as Sample A in this paper) the size of Ag clusters increased to approximately $6 \mathrm{~nm}$ [see Fig. 2(b)]. The cluster density increased significantly. The structure of the Ag clusters was first characterized by HRTEM [Fig. 2(e)]. As shown in the inset in Fig. 2(e) (Sample A), the Ag clusters are crystalline with clear facets and the lattice-plane spacing measured from the HRTEM image matched the $d$-spacing of (111) plane of the Ag face-center-cubic structure. However, the STM image of the same sample revealed an average size of the clusters smaller than that observed by HRTEM. Obviously this is because HRTEM is not able to show a clear contrast of very thin layer of $\mathrm{Ag}$ atoms distributed around the edges of the clusters. We use L (the average edge-to-edge distance between clusters) to describe the separation between the clusters. L can reflect the perfect graphene area unaffected by the clusters. The "puddle-like" Ag clusters can be seen more clearly in the highresolution STM image in Fig. 2(f) (Sample B, medium coating for 5 seconds). The actual affected area (marked by the arrows)
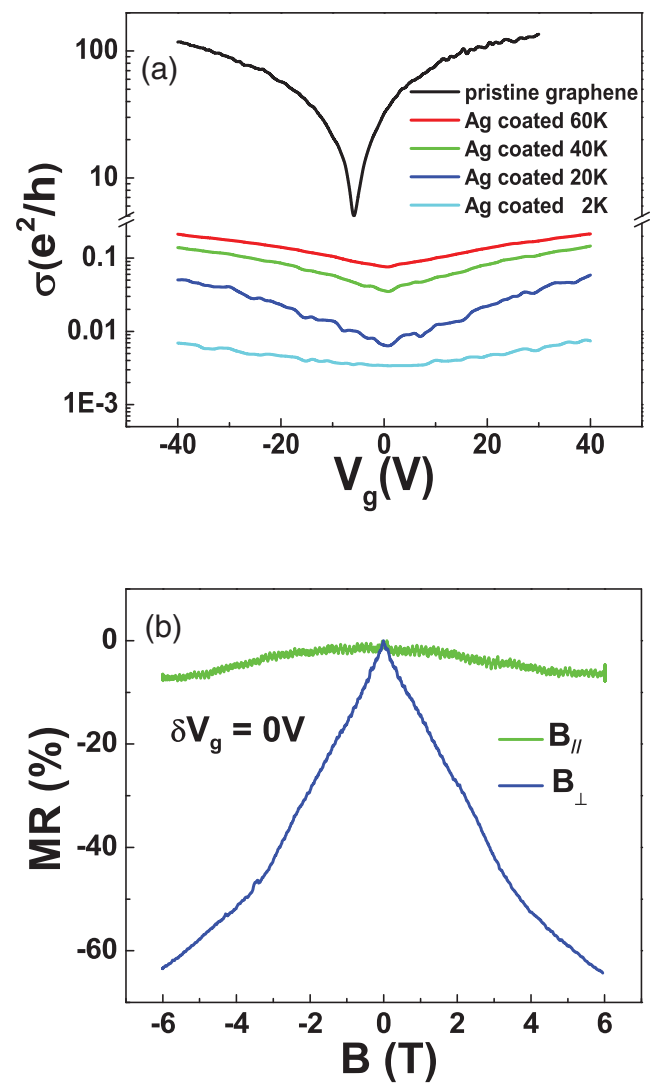

on graphene surrounding a cluster may be slightly larger than the cluster size. According to the STM images, the average separation L between Ag clusters in Sample A [Fig. 2(b)] is about 7-8 nm. For medium (Sample B) and heavily (Sample C, 10 second coating) decorated samples, the average separations were about 3-4 $\mathrm{nm}$ and $2-3 \mathrm{~nm}$, respectively.

Ag clusters caused obvious changes to the Raman spectra (excitation wavelength $\lambda=514 \mathrm{~nm}$ ). Compared to the pristine graphene devices, the most significant change is the appearance of the D-band at $1350 \mathrm{~cm}^{-1}$ [Fig. 2(d)], which has been assigned to the breathing mode of $s p^{2}$ for phonons with wavevectors near the $\left(\mathrm{K}, \mathrm{K}^{\prime}\right)$ points, activated by disorder. It has been widely accepted that the D-band involves a double resonance process and a disorder-induced mode. ${ }^{26}$ For comparison we used e-beam evaporation to deposit $\mathrm{Ag}, \mathrm{Au}$, and $\mathrm{Cr}$ clusters on single-layer graphene and observed similar D-band feature. However, the intensity of the D-band is weaker [see Fig. 2(d)] than that in the samples decorated by DC-plasma deposition. Obviously metal clusters attached on the graphene surface (assisted by DC plasma) have a strong interaction with graphene and may break the symmetry of the honeycomb crystal structure. In previous studies it has been suggested that D-bands appeared in the Raman scattering of folded graphene ${ }^{27}$ were attributable to the symmetry breaking of graphene structure. The defect spacing can be estimated roughly using the empirical formula $\left.L_{a}(\mathrm{~nm})=\left[2.4 \times 10^{-10} \mathrm{~nm}^{-3}\right] \lambda^{4}\left(I_{D} / I_{G}\right)^{-1}\right]$, which correlates
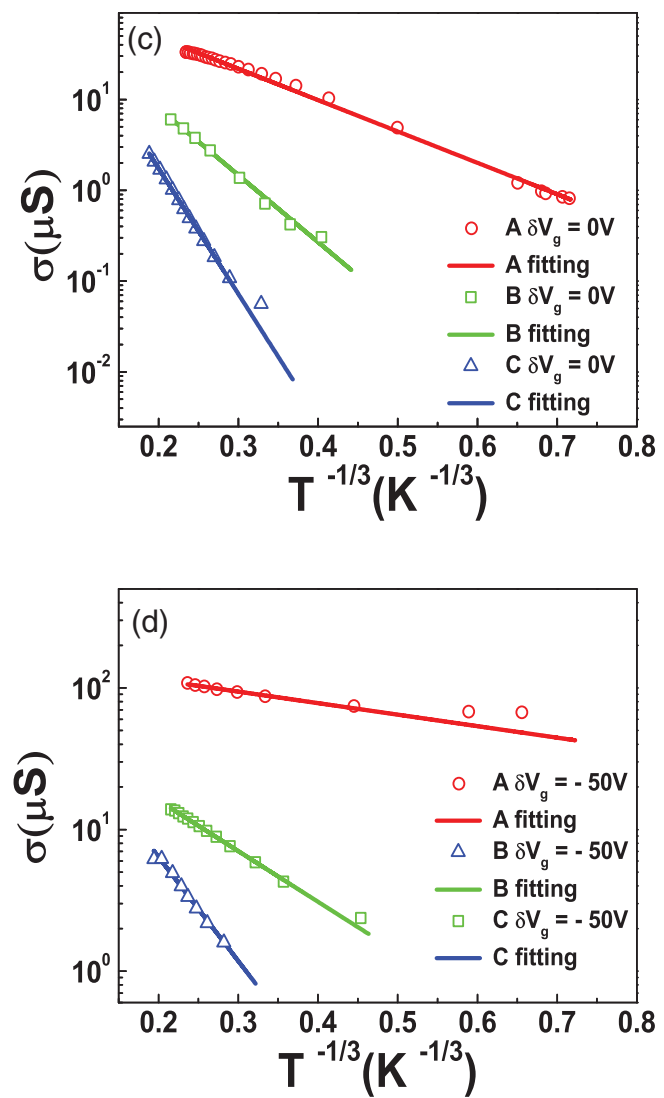

FIG. 3. (Color online) (a) Dramatic decrease of the conductance of Ag-decorated graphene (Sample C, heavily coated) at low temperature. (b) The MR effect obtained from Sample A with the magnetic field parallel (green) and perpendicular (blue) to the device surface. (c), (d) The temperature dependences of the conductivity plotted as $\sigma$ versus $T^{-1 / 3}$ in log scale for different gate voltages. Solid lines represent the fitting results based on Mott's law. 
TABLE I. Table 1. Localization length, density of state, and mobility in Ag-decorated graphene.

\begin{tabular}{|c|c|c|c|c|c|}
\hline Sample & $\begin{array}{c}\xi_{\mathrm{VRH}}(\mathrm{nm}) \\
\left(\delta V_{g}=0 \mathrm{~V}\right)\end{array}$ & $\begin{array}{c}\rho\left(E_{\mathrm{F}}\right)\left(10^{16} \mathrm{eV}^{-1} \mathrm{~m}^{-2}\right) \\
\left(\delta V_{g}=0 \mathrm{~V}\right)\end{array}$ & $\begin{array}{c}\xi_{\mathrm{VRH}}(\mathrm{nm}) \\
\left(\delta V_{g}=-50 \mathrm{~V}\right)\end{array}$ & $\begin{array}{c}\rho\left(E_{\mathrm{F}}\right)\left(10^{16} \mathrm{eV}^{-1} \mathrm{~m}^{-2}\right) \\
\left(\delta V_{g}=-50 \mathrm{~V}\right)\end{array}$ & $\mu\left(\mathrm{cm}^{2} \mathrm{~V}^{-1} \mathrm{~s}^{-1}\right)$ \\
\hline A & $90 \pm 3$ & $2.5 \pm 0.5$ & $420 \pm 20$ & $7 \pm 3$ & $250 \pm 30$ \\
\hline B & $22 \pm 5$ & $6 \pm 2$ & $50 \pm 15$ & $11 \pm 4$ & $140 \pm 30$ \\
\hline $\mathrm{C}$ & $6 \pm 2$ & $14 \pm 4$ & $13 \pm 3$ & $25 \pm 5$ & $70 \pm 20$ \\
\hline
\end{tabular}

the intensity of the D-band $\left(\mathrm{I}_{\mathrm{D}}\right)$ and $\mathrm{G}$-band $\left(\mathrm{I}_{\mathrm{G}}\right) \cdot{ }^{28}$ For the lightly decorated Sample A, $L_{a}$ is estimated to be approximately $24 \mathrm{~nm}$, which is noted to be somewhat larger than the average distance of $\mathrm{Ag}$ clusters observed from STM images. For the medium decorated Sample B, $L_{a}$ is $7 \mathrm{~nm}$, indicating a high density of disorder (attributable to interaction between $\mathrm{Ag}$ and graphene) on the graphene device. The Ag clusters resulted in significant changes in the graphene conductivity. The resistance of the devices was observed to increase by about one order of magnitude at room temperature (a maximum of three orders of increase has been observed) and the mobility was largely reduced (see Table I) after Ag decoration. As shown in Fig. 3(a), the Dirac point shifted slightly and the conductivity became very sensitive to temperature. At low temperatures (down to $1.8 \mathrm{~K}$ ), the conductivity of Ag-decorated devices decreased drastically, and the heavily decorated devices often behaved as insulators. Figures 3(c) and 3(d) illustrate the change of the resistivity versus temperature for different Ag-coating densities recorded at $V_{g}=0 \mathrm{~V}$ and $-50 \mathrm{~V}$, respectively. Combining the data shown in Figs. 3(a), 3(c), and 3(d), it seems that Ag clusters, deposited using DC plasma, introduced strong disorder-scattering centers and caused localized states. Therefore, the transport of the graphene devices at low temperatures followed the VRH conductance model. ${ }^{29,30}$ For Mott's VRH process an electron can hop from one localized site to another when receiving energy, and the resistivity in the Miller-Abrahams network may be expressed by

$$
\rho \propto \exp \left(\frac{2 r_{i j}}{\xi}+\frac{\varepsilon_{i j}}{k T}\right),
$$

where $r_{i j}$ is the hop length between site $i$ and $j$, and $\xi$ the localization length. By using the argument of critical conduction path, ${ }^{31}$ it can be shown that the temperature dependence of the resistivity is dependent on the density of states at the Fermi level. For a constant density of states at the Fermi level, the resistivity is described as $\rho(T) \propto \exp \left(\frac{T_{0}}{T}\right)^{\frac{1}{1+d}}$, where $d=2$ for a 2D system. ${ }^{29,31}$ Figures 3(c) and 3(d) clearly display a $\sigma \propto \exp \left(\frac{T_{0}}{T}\right)^{-\frac{1}{3}}$ behavior that is consistent with $2 \mathrm{D}$ VRH conduction. Here $T_{0}$ is noted to be inversely proportional to the density of states.

To understand the transport properties of the Ag-decorated graphene, we have systematically investigated the MR effects in the presence of a magnetic field perpendicular to the graphene layer. In contrast to pristine graphene the metal cluster-decorated graphene devices displayed large negative MR effects [the blue curve in Fig. 3(b)]. Their MRs were gatevoltage dependent. The maximum MR effect was achieved with the lowest carrier density when $V_{g}$ was approaching the Dirac point. To verify the $2 \mathrm{D}$ quantum interferences of localization effects in Ag decorated samples, we also performed MR measurement with a magnetic field parallel to the graphene surface. As shown in Fig. 3(b) the negative MR effect was much weaker than that in the same sample recorded with a perpendicular magnetic field. The MR effect at $6 \mathrm{~T}$ was approximately $-10 \%$, while under the perpendicular magnetic field the MR effect was approximately $-70 \%$. This strong magnetic field direction dependence of the negative MR effect indicated a major localization effect which might arise from the quantum interference between the charge carrier paths.

In order to clarify the effect of DC plasma in the deposition of the Ag clusters, we have deposited Ag clusters by electronbeam evaporation. The nominal thickness of Ag clusters deposited was $2 \AA$, and the cluster size was similar to that deposited by the DC plasma. As shown in Fig. 4(a) the conductivity of the graphene sample decreased slightly and the Dirac points shifted slightly toward left compared to the pristine sample. These results were consistent with that reported previously. ${ }^{15,32,33}$ Therefore, the metal clusters formed on the graphene surface by electron-beam evaporation should be simple physical absorption. We have also carried out investigation of the DC-plasma effect without deposition of Ag clusters. We used a plasma cleaner (PDC-002, Harrick Plasma) at a power of $7 \mathrm{~W}$ (slightly stronger than that used for Ag decoration) for $5 \mathrm{~s}$ with the plasma directly exposed to the graphene surface. Again the change of the graphene conductivity [Fig. 4(b)] was not significant in comparison with the results of plasma-assisted Ag deposition as shown
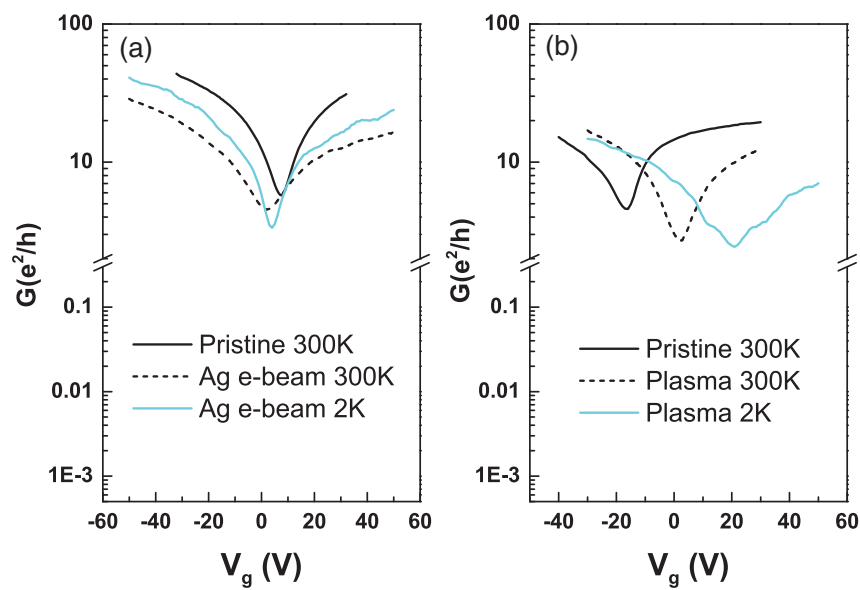

FIG. 4. (Color online) The slight changes of the conductivity and Dirac points of graphene samples after (a) deposition of Ag clusters by the electron-beam evaporation (without DC plasma) and (b) the DC-plasma treatment (without metal cluster deposition). 

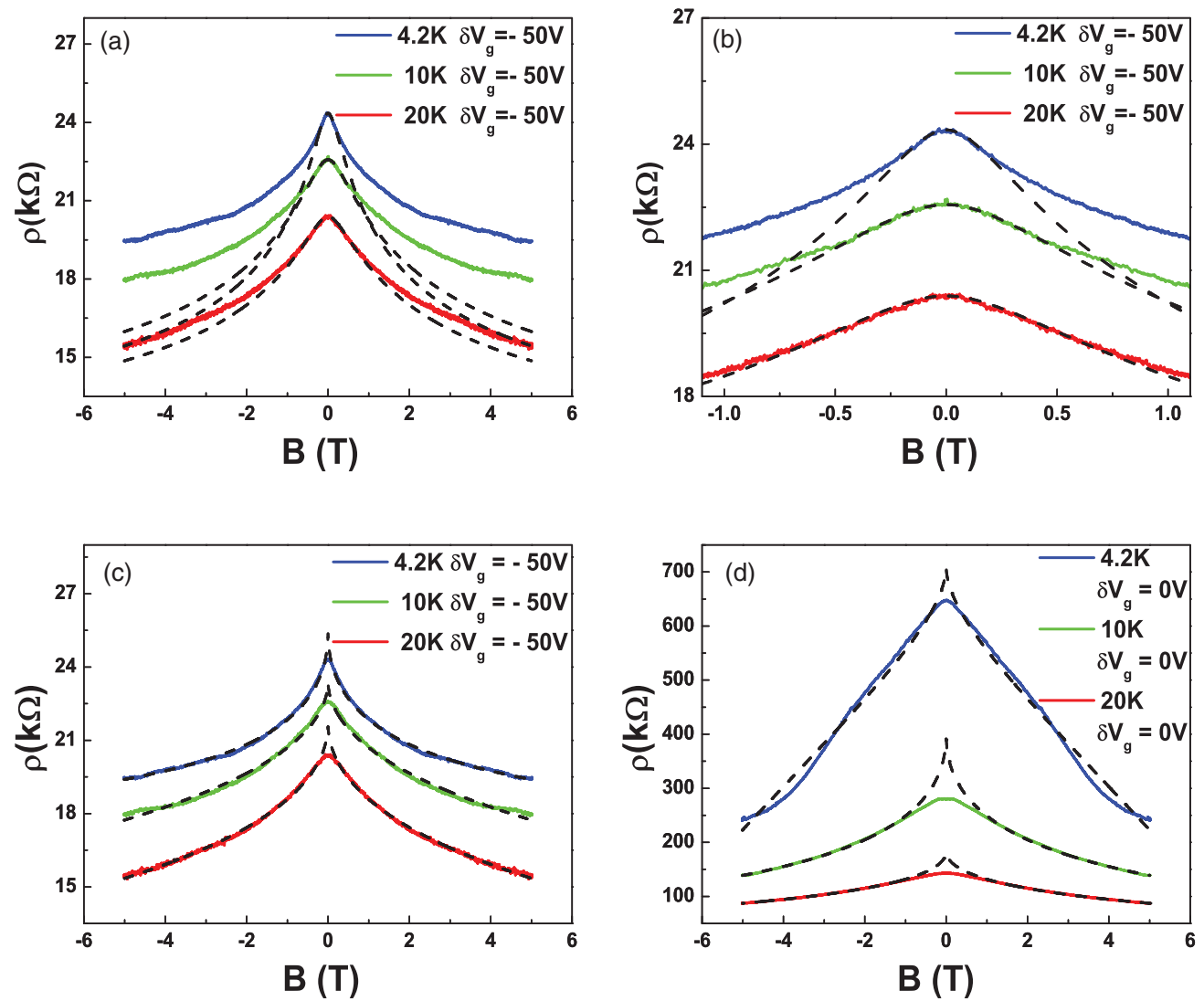

FIG. 5. (Color online) (a) MR curves of sample A measured at $4.2 \mathrm{~K}, 10 \mathrm{~K}$, and $20 \mathrm{~K}$ for $V_{g}=-50 \mathrm{~V}$. The dashed lines are fitting results based on the E. McCann description of WL model. (b) Enlarged figure showing the fitting near zero magnetic field. (c) Dashed lines are fitting results based on $\rho \propto \exp \left(-B^{1 / 2}\right)$. (d) The MR data measured at the Dirac point of the graphene device show a large deviation from $\rho \propto \exp \left(-B^{1 / 2}\right)$.

in Fig. 3(a). The Dirac points shifted slightly toward right compared to that of the pristine sample. The Raman spectrum recorded from the plasma-treated sample did not show obvious increase of the D-band [see Fig. 2(d)]. Obviously few defects were generated by the DC plasma for such a short period. Therefore, neither the DC plasma nor simple Ag-cluster deposition could individually induce the sources of such a strong scattering [see Fig. 3(a)] in graphene as observed by DC plasma-assisted Ag decoration. The MR measurements for these two samples did not show strong negative MR effects. We believe that the DC plasma-assisted Ag decoration had activated $\mathrm{Ag}$ and carbon atoms in graphene, which resulted in a strong interaction or chemical bonding between $\mathrm{Ag}$ and carbon atoms. Such activated decoration processes have been reported in other graphitic materials in previous research. ${ }^{34,35}$ In this case Ag clusters strongly bond with carbon atoms and largely enhance the scatterings of electron transport in graphene. To generate Ag clusters, a DC voltage (about 450-500 V) is applied to the Ag target. In a general sputtering process the metal clusters leave from the target with a translational kinetic energy of approximately $1-10 \mathrm{eV} .{ }^{36}$ For a DC voltage of about $500 \mathrm{~V}$ applied to a metal target, the main kinetic energy of the metal clusters leaving the target surface is about $3 \mathrm{eV}$ (see Fig. 10 in Ref. 36). The real kinetic energy of the clusters when they reach the sample surface is further reduced because of the collision between clusters. Therefore, the incident energy of sputtered metal clusters when they reach graphene is much smaller than $50 \mathrm{eV}$, at which the metal clusters may cause knock-on damage to graphene. But we also want to add that owing to such strong interaction, destruction of the pristine graphene structure at the contact regions of metal clusters cannot be ruled out at this point.

For slightly Ag-decorated samples we expect that both intervalley and intravalley scattering rates are greatly increased. This is not only supported by the fact that the D-band appeared in the Raman scattering but also revealed by its lowtemperature transport behavior. Because the transport behavior of the samples deviates from that of pure graphene, we used different models to fit the experimental data. First to estimate the scattering rates, we consider weak-point disorder for the intervalley scattering time $\tau_{\text {inter }}=\sigma_{s} \cdot h /\left(2 e^{2} \cdot v_{F} \sqrt{\pi n}\right)$ and the charged impurity for the intravalley scattering time $\tau_{\text {intra }}=h /\left(2 e^{2} \cdot v_{F} \sqrt{\pi}\right) \cdot \mu \cdot \sqrt{n}$. The measured conductivity $\sigma_{\text {meas }}\left(V_{g}\right)$ should consist of long-range scattering and short-range scattering components, i.e., $\sigma_{\text {meas }}^{-1}=(n e \mu)^{-1}+$ $\left(\sigma_{s}\right)^{-1} .37$ For Sample A we obtained $\sigma_{s}=2.2 \times 10^{-4} S$ and $\mu=250 \mathrm{~cm}^{2} \mathrm{~V}^{-1} \mathrm{~s}^{-1}$. We therefore determined that the upper bound of the intervalley and intravalley scattering time is approximately $15 \mathrm{fs}$. In the Boltzmann theory the graphene conductivity is given by the Einstein relation $\sigma=e^{2} D \rho\left(E_{F}\right)$, where the diffusion constant is described by $D=\frac{1}{2} v_{F}^{2} \tau$, with $\tau$ being the relaxation time and $\rho\left(E_{\mathrm{F}}\right)$ the density of states. Several different scattering mechanisms involving local-point defects and long range Coulomb scattering ${ }^{10,24,38,39}$ can be 
Sample B
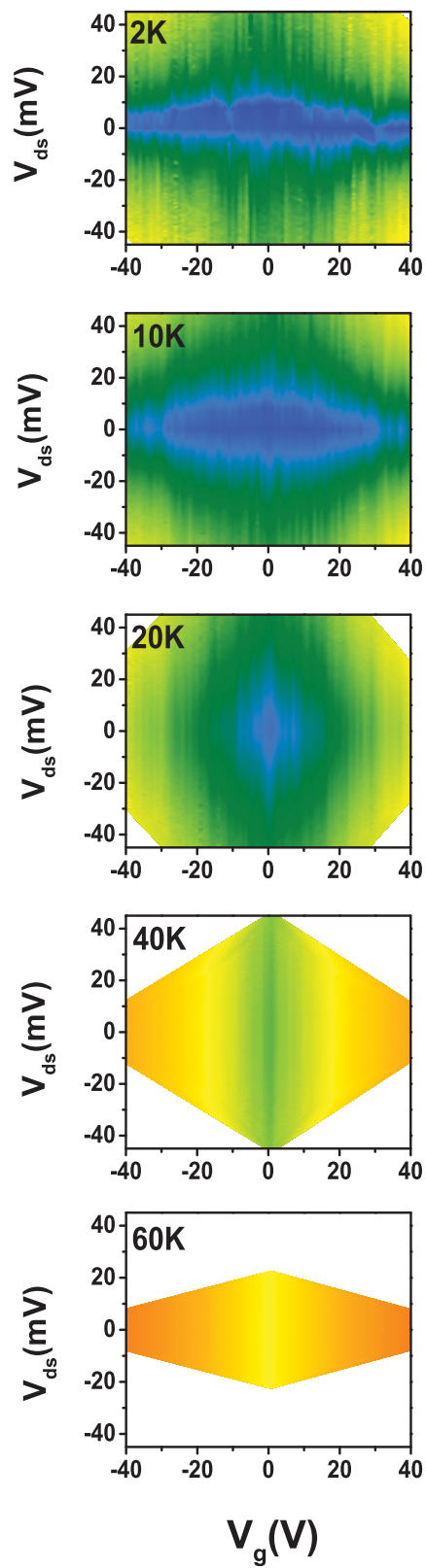

Sample C
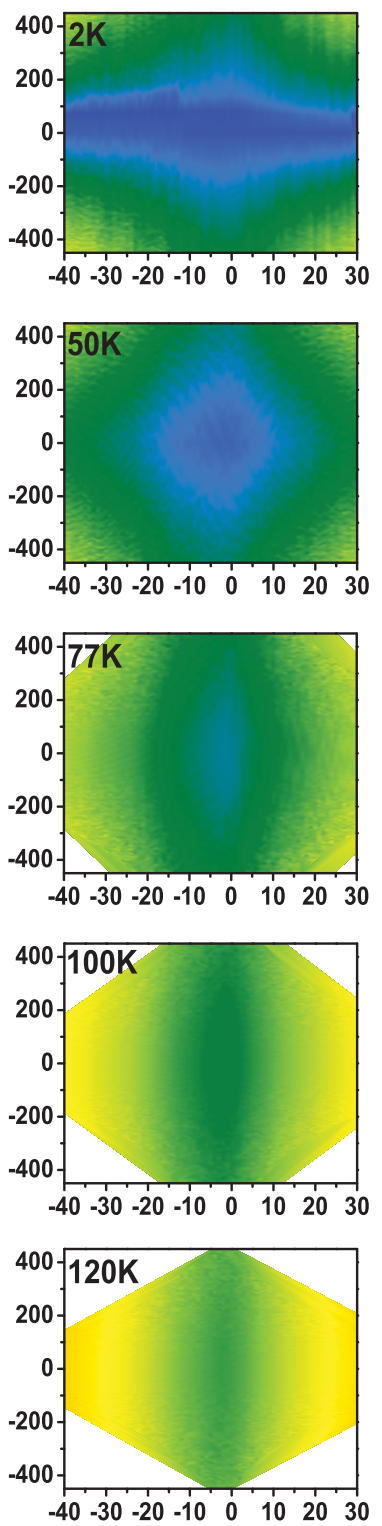

$\mathrm{V}_{\mathrm{g}}(\mathrm{V})$
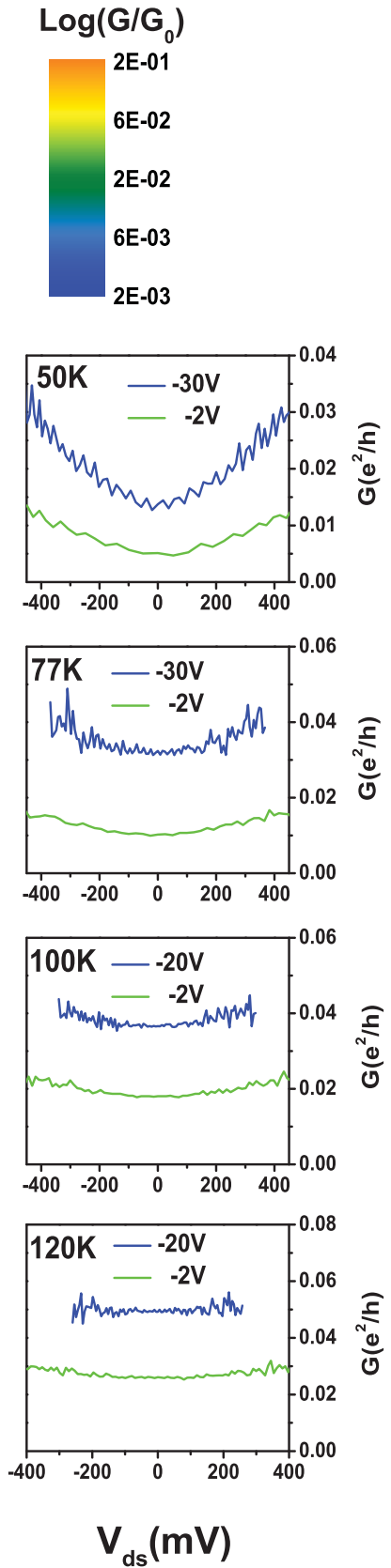

FIG. 6. (Color online) The temperature dependence of the differential conductance under different biases and gate voltages for Sample B and $\mathrm{C}$, respectively. The right column shows the oscillation of differential conductance extracted at different gate voltages.

used to determine the relaxation time $\tau$ in graphene. For graphene with disorders, McCann et al. ${ }^{7}$ have obtained a formula for WL correction to the MR as follows,

$$
\begin{aligned}
\rho(B)= & -\frac{e^{2} \rho^{2}}{\pi h}\left[F\left(\frac{\tau_{B}^{-1}}{\tau_{\phi}^{-1}}\right)-F\left(\frac{\tau_{B}^{-1}}{\tau_{\phi}^{-1}+2 \tau_{i}^{-1}}\right)\right. \\
& \left.-2 F\left(\frac{\tau_{B}^{-1}}{\tau_{\phi}^{-1}+\tau_{i}^{-1}+\tau_{*}^{-1}}\right)\right] .
\end{aligned}
$$

Here $F(z)=\ln z+\varphi\left(\frac{1}{2}+\frac{1}{z}\right), \varphi$ is the digamma function $\tau_{B}^{-1}=4 e D B / \hbar . \tau_{\phi}$ is the phase coherence time; $\tau_{i}$ and $\tau_{*}$ are the intervalley and intravalley scattering time, respectively. The first term in Eq. (2) is responsible for WL effect, and the other two terms describe AL. In the metallic regime where the system is far away from the Dirac point, the formula obtained by McCann for WL is still valid. Therefore, the experimental data may be fitted by the formula. By fitting the experimental MR results, we found that in the low external magnetic field regime, the fitting curves can qualitatively match the experimental data [see Figs. 5(a) and 5(b)]. For Sample A we extracted the phase-coherence length $L_{\phi}=$ $\left(D \tau_{\phi}\right)^{1 / 2}=31 \mathrm{~nm}$ with a phase-coherence time $\tau_{\phi}=650 \mathrm{fs}$ (at $2.5 \mathrm{~K}$ ). We note that although the model can fit the experimental MR data in the low external magnetic field ( $B<500 \mathrm{Gs}$ ), there is a large deviation between the MR data and the fitting curves at the high magnetic field regime [see Fig. 5(a)]. 
The observation of VRH behavior suggests SL. It is known that in the weak magnetic field regime where the magnetic length is larger than the localization length, there can be a negative MR. ${ }^{40}$ Based on a numerical study of the orbital mechanism, Zhao et al. showed that the magnetoconductance $L=\left\langle\ln \frac{\sigma_{i j(H)}}{\sigma_{i j(0)}}\right\rangle$ depended linearly on the distance $(r)$ between the initial $(i)$ and final $(j)$ sites as well as on the magnetic length $\left(L_{H}\right),{ }^{41}$

$$
L=\gamma \frac{r}{L_{H}},
$$

where $L_{H}=\left(\phi_{0} / H\right)^{1 / 2}$ and $\gamma$ is a numerical coefficient. This linear dependence corresponds to a magnetic field correction to the localization length. ${ }^{42}$ Therefore, the field dependence of the MR was suggested to be $\ln [R(H) / R(0)] \propto-H^{1 / 2}$ [equivalent to $\left.\rho \propto \exp \left(-B^{1 / 2}\right)\right]$ in the weak magnetic field limit. ${ }^{41,43}$ In Fig. 5(c) we show the fitting curves of the MR experimental data versus the magnetic field. The dependence of the MR on $-B^{1 / 2}$ agrees with the orbital mechanism of $2 \mathrm{D} V R H$ very well. As shown in Fig. 5(d), however, the MR data recorded at the Dirac point cannot be fitted well using $\rho \propto \exp \left(-B^{1 / 2}\right)$. The drastic increase in resistance by orders of magnitude implies a decrease in the localization length. It follows that with the decrease in the localization length the effect of the magnetic field would decrease as well, as the cross-sectional area of the localized state that is sensitive to the magnetic field becomes negligible. This is exactly what was observed in our MR measurements of the heavily decorated samples. By estimating the electron-diffusion constant, we obtained the changes of the density of states versus gate voltages. Then we estimated the localization length based on the 2D VRH model, ${ }^{44}$

$$
\xi_{\mathrm{VRH}}=\sqrt{\frac{13.8}{k_{B} \rho(E) T_{0}}},
$$

where $k_{\mathrm{B}}$ is the Boltzmann constant. For Sample $\mathrm{A}$ the localization length at the gate voltage far away from the Dirac point $\left(\delta V_{g}=-50 \mathrm{~V}\right)$ is approximately $420 \mathrm{~nm}$, about 50 times of the separation distance of the Ag clusters. At the Dirac point the localization length is approximately $90 \mathrm{~nm}$ (see Table I). For Samples B and C the localization lengths are significantly decreased compared to Sample A. With increasing density of Ag clusters we observed an increase in the density of states. We estimated the density of states by first calculating the electron mobility and diffusion constant. The density of states is then obtained according to the Einstein relation $\sigma=e^{2} D \rho\left(E_{F}\right)$. This estimation was done using the experimental data measured near room temperature in order to minimize the influence of the SL effect at low temperatures. Because the uncertainty of the estimated density of states is large we show the localization lengths [obtained according to Eq. (4)] listed in Table I accompanied by their respective error bars. The density of states obtained for Sample A at $300 \mathrm{~K}$ is approximately $2 \times 10^{16} \mathrm{eV}^{-1} \mathrm{~m}^{-2}$ (close to the Dirac point). With a gate voltage of $-50 \mathrm{~V}$ away from the Dirac point, the density of states increases to $7 \times 10^{16} \mathrm{eV}^{-1} \mathrm{~m}^{-2}$. We note that the density of states estimated for Ag-decorated graphene is slightly higher than those measured from pristine graphene. ${ }^{45}$

We have measured the differential conductance of the samples under different biases and gate voltages. Figure 6 qualitatively demonstrates the mapping of differential conductance of mild and heavily Ag-decorated devices for different temperatures. Because the samples are in the SL regime, no obvious MR effect was observed under a magnetic field up to $6 \mathrm{~T}$. In Fig. 6 oscillations in the differential conductance become pronounced when the sample temperature is below $100 \mathrm{~K}$. The oscillation is temperature dependent and very obvious during variation of drain-source voltage $\left(V_{\mathrm{ds}}\right)$ for a fixed gate voltage $\left(V_{g}\right)$ (see the right-side column). There seems to be no rigorous periodicity of the oscillation. The right column shows the variation of the differential conductances versus $V_{\mathrm{ds}}$ for certain gate voltages. The oscillation of the differential conductance versus $V_{\mathrm{ds}}$ is clearly visible for $50 \mathrm{~K}$. For high temperatures $(>120 \mathrm{~K})$ the oscillation gradually disappears.

We consider the oscillation phenomenon, or the apparent changes of the slope on the $I-V$ characteristic, being attributable to the fluctuations in 2D-hopping conductance. This is because the sample length is short (approximately $2.4 \mu \mathrm{m}$ ), and different bias voltage can pick out different initial states and lead to different hopping conduction paths. Mesoscopic fluctuation effects have been observed in 1D-46 and 2D-semiconductor field-effect transistors (FET) structures ${ }^{47}$ that have the same order of dimension in which the differential conductance oscillates when varying the number of the electrons in the FET channel through the gate voltage. The oscillations have been explained on the basis of a model of a "main" hop. ${ }^{48-50}$ As for the samples in the hopping conductance regime, their conductance can be described as the sum of the conductance of various paths, which consist of chains of hops. The conductance of the sample is determined by one of the best conducting chains because of the exponentially spreading in the resistances of the hops. The oscillation of the conductance is therefore attributed to the redistribution of the voltage among the resistances of the hops of the leading chain. ${ }^{48}$

\section{CONCLUSION}

The lattice disorder introduced by noble-metal cluster decoration, using DC plasma deposition, largely changed the transport properties of graphene. A strong interaction between metal clusters and graphene has been evidenced by micro-Raman scattering. The metal-decorated graphene transformed from WL to SL by increasing the density of the metal clusters. At low temperature the heavily Ag-decorated graphene behaved as an insulator, and its transport property was in accordance with the 2D VRH mechanism. The large MR effect observed in the samples showed obvious dependence of $\rho \propto \exp \left(-B^{1 / 2}\right)$, which agreed with the orbital mechanism of 2D VRH fairly well.

\section{ACKNOWLEDGMENTS}

The authors are grateful for helpful discussions with C. T. Chan and Q. C. Zhang. Financial support from the Research Grants Council of Hong Kong (Project Nos. HKUST9/CRF/08, 603408, 604009) and technical support of the Raith-HKUST Nanotechnology Laboratory for the electron-beam lithography facility (Project No. SEG_HKUST08) are hereby acknowledged. 
*phwang@ust.hk

${ }^{1}$ K. S. Novoselov, A. K. Geim, S. V. Morozov, D. Jiang, Y. Zhang, S. V. Dubonos, I. V. Grigorieva, and A. A. Firsov, Science 306, 666 (2004).

${ }^{2}$ S. Hikami, A. I. Larkin, and Y. Nagaoka, Prog. Theor. Phys. 63, 707 (1980).

${ }^{3}$ D. P. DiVincenzo and E. J. Mele, Phys. Rev. B 29, 1685 (1984).

${ }^{4}$ G. W. Semenoff, Phys. Rev. Lett. 53, 2449 (1984).

${ }^{5}$ H. Suzuura and T. Ando, Phys. Rev. Lett. 89, 266603 (2002).

${ }^{6}$ D. V. Khveshchenko, Phys. Rev. Lett. 97, 036802 (2006).

${ }^{7}$ E. McCann, K. Kechedzhi, V. I. Fal'ko, H. Suzuura, T. Ando, and B. L. Altshuler, Phys. Rev. Lett. 97, 146805 (2006).

${ }^{8}$ I. L. Aleiner and K. B. Efetov, Phys. Rev. Lett. 97, 236801 (2006).

${ }^{9}$ A. Altland, Phys. Rev. Lett. 97, 236802 (2006).

${ }^{10}$ P. M. Ostrovsky, I. V. Gornyi, and A. D. Mirlin, Phys. Rev. B 74, 235443 (2006).

${ }^{11}$ A. F. Morpurgo and F. Guinea, Phys. Rev. Lett. 97, 196804 (2006).

${ }^{12}$ F. V. Tikhonenko, D. W. Horsell, R. V. Gorbachev, and A. K. Savchenko, Phys. Rev. Lett. 100, 056802 (2008).

${ }^{13}$ F. V. Tikhonenko, A. A. Kozikov, A. K. Savchenko, and R. V. Gorbachev, Phys. Rev. Lett. 103, 226801 (2009).

${ }^{14}$ Y. F. Chen, M. H. Bae, C. Chialvo, T. Dirks, A. Bezryadin, and N. Mason, J. Phys. Condens. Matter 22, 205301 (2010).

${ }^{15}$ J. H. Chen, C. Jang, S. Adam, M. S. Fuhrer, E. D. Williams, and M. Ishigami, Nat. Phys. 4, 377 (2008).

${ }^{16}$ J. H. Chen, W. G. Cullen, C. Jang, M. S. Fuhrer, and E. D. Williams, Phys. Rev. Lett. 102, 236805 (2009).

${ }^{17}$ R. Muszynski, B. Seger, and P. V. Kamat, J. Phys. Chem. C 112, 5263 (2008).

${ }^{18}$ G. Williams, B. Seger, and P. V. Kamat, ACS Nano 2, 1487 (2008).

${ }^{19}$ D. C. Elias, R. R. Nair, T. M. G. Mohiuddin, S. V. Morozov, P. Blake, M. P. Halsall, A. C. Ferrari, D. W. Boukhvalov, M. I. Katsnelson, A. K. Geim, and K. S. Novoselov, Science 323, 610 (2009).

${ }^{20}$ J. Moser, H. Tao, S. Roche, F. Alzina, C. M. Sotomayor Torres, and A. Bachtold, Phys. Rev. B 81, 205445 (2010).

${ }^{21}$ R. Balog, B. Jorgensen, L. Nilsson, M. Andersen, E. Rienks, M. Bianchi, M. Fanetti, E. Laegsgaard, A. Baraldi, S. Lizzit, Z. Sljivancanin, F. Besenbacher, B. Hammer, T. G. Pedersen, P. Hofmann, and L. Hornekaer, Nat. Mater. 9, 315 (2010).

${ }^{22}$ T. Ando, J. Phys. Soc. Jpn. 75, 074716 (2006).

${ }^{23}$ K. Nomura and A. H. MacDonald, Phys. Rev. Lett. 98, 076602 (2007).

${ }^{24}$ S. Adam, E. H. Hwang, V. M. Galitski, and S. Das Sarma, Proc. Natl. Acad. Sci. USA 104, 18392 (2007).

${ }^{25}$ M. Trushin and J. Schliemann, Phys. Rev. Lett. 99, 216602 (2007).

${ }^{26}$ M. S. Dresselhaus, G. Dresselhaus, R. Saito, and A. Jorio, Phys. Rep. 409, 47 (2005).

${ }^{27}$ A. K. Gupta, C. Nisoli, P. E. Lammert, V. H. Crespi, and P. C. Eklund, J. Phys. Condens. Matter 22, 334205 (2010).
${ }^{28}$ L. G. Cancado, K. Takai, T. Enoki, M. Endo, Y. A. Kim, H. Mizusaki, A. Jorio, L. N. Coelho, R. Magalhaes-Paniago, and M. A. Pimenta, Appl. Phys. Lett. 88, 163106 (2006).

${ }^{29}$ N. F. Mott and E. A. Davis, in Electronic Processes in Noncrystalline Materials (Clarendon Press, Oxford, 1979), p. 32.

${ }^{30}$ C. Gomez-Navarro, R. T. Weitz, A. M. Bittner, M. Scolari, A. Mews, M. Burghard, and K. Kern, Nano Lett. 7, 3499 (2007).

${ }^{31}$ V. Ambegaokar, B. I. Halperin, and J. S. Langer, Phys. Rev. B 4, 2612 (1971).

${ }^{32}$ K. Pi, K. M. McCreary, W. Bao, W. Han, Y. F. Chiang, Y. Li, S. W. Tsai, C. N. Lau, and R. K. Kawakami, Phys. Rev. B 80, 075406 (2009).

${ }^{33}$ K. M. McCreary, K. Pi, A. G. Swartz, W. Han, W. Bao, C. N. Lau, F. Guinea, M. I. Katsnelson, and R. K. Kawakami, Phys. Rev. B 81, 115453 (2010)

${ }^{34}$ Q. Chen, L. Dai, M. Gao, S. Huang, and A. Mau, J. Phys. Chem. B 105, 618 (2000).

${ }^{35}$ J. F. Friedrich, S. Wettmarshausen, S. Hanelt, R. Mach, R. Mix, E. B. Zeynalov, and A. Meyer-Plath, Carbon 48, 3884 (2010).

${ }^{36}$ J. J. Cuomo, S. M. Rossnagel, and H. R. Kaufman, in Handbook of Ion Beam Processing Technology_Principles, Deposition, Film Modification and Synthesis (Noyes Publications, New Jersey, 1989), p. 91.

${ }^{37}$ S. V. Morozov, K. S. Novoselov, M. I. Katsnelson, F. Schedin, D. C. Elias, J. A. Jaszczak, and A. K. Geim, Phys. Rev. Lett. 100, 016602 (2008).

${ }^{38}$ N. M. R. Peres, F. Guinea, and A. H. Castro Neto, Phys. Rev. B 73, 125411 (2006).

${ }^{39}$ T. Stauber, N. M. R. Peres, and F. Guinea, Phys. Rev. B 76, 205423 (2007).

${ }^{40}$ B. I. Shklovskii and B. Z. Spivak, in Hopping Transport in Solids, edited by M. Pollak and B. I. Shklovskii (North-Holland, Amsterdam, 1990), p. 271.

${ }^{41}$ H. L. Zhao, B. Z. Spivak, M. P. Gelfand, and S. Feng, Phys. Rev. B 44, 10760 (1991).

${ }^{42}$ E. Medina, M. Kardar, Y. Shapir, and X. R. Wang, Phys. Rev. Lett. 64, 1816 (1990).

${ }^{43}$ H. W. Jiang, C. E. Johnson, and K. L. Wang, Phys. Rev. B 46, 12830 (1992).

${ }^{44}$ B. I. Shklovskii and A. L. Efros, Electronic Properties of Doped Semiconductors (Springer-Verlag, Berlin, 1984), p. 206.

${ }^{45}$ S. Droscher, P. Roulleau, F. Molitor, P. Studerus, C. Stampfer, K. Ensslin, and T. Ihn, Appl. Phys. Lett. 96, 152104 (2010).

${ }^{46}$ R. A. Webb, A. Hartstein, J. J. Wainer, and A. B. Fowler, Phys. Rev. Lett. 54, 1577 (1985).

${ }^{47}$ K. Aoki, Y. Okuyama, T. Kobayashi, and K. Yamamoto, J. Phys. C. 12, 647 (1979).

${ }^{48}$ M. Pollak and I. Riess, J. Phys. C. 9, 2339 (1976).

${ }^{49}$ P. A. Lee, Phys. Rev. Lett. 53, 2042 (1984).

${ }^{50}$ A. O. Orlov and A. K. Savchenko, Jetp Lett. 47, 470 (1988). 